\title{
The AN703 antibody recognizes Dictyostelium discoideum PDI by immunofluorescence
}

\author{
Anna Marchetti
}

Geneva Antibody Facility, Faculty of Medicine, University of Geneva, 1 rue Michel Servet, CH-1211, Geneva, Switzerland

\begin{abstract}
The AN703 antibody, derived from the 221-42-1 hybridoma, detects PDI-containing endoplasmic reticulum of Dictyostelium discoideum by immunofluorescence.

Introduction

Protein disulfide isomerase (PDI, DDB_G0276141, UniProt Q86IA3) is a protein present in the endoplasmic reticulum (ER) in D. discoideum, and it is recognized by the 221-42-1 monoclonal antibody (Monnat et al., 1997). Here we describe the ability of the AN703 antibody, a single chain fragment ( $\mathrm{scFv}$ ) derived from the 221-42-1 hybridoma, to label the endoplasmic reticulum by immunofluorescence.
\end{abstract}

\section{Materials \& Methods}

\begin{abstract}
Antibodies: The ABCD_AN703 antibody (ABCD nomenclature, http://web.expasy.org/abcd/) was produced by the Geneva Antibody Facility (http://www.unige.ch/ medecine/antibodies/) as mini-antibody with the antigenbinding $\mathrm{scF}_{\mathrm{V}}$ fused to a mouse IgG2A Fc. The synthesized scFv sequence (GeneArt, Invitrogen) corresponds to the sequence of the variable regions joined by a peptide linker (GGGGS) $)_{3}$. The sequencing of the 221-42-1 hybridoma was performed by the Geneva Antibody Facility. HEK293 suspension cells (growing in FreeStyle ${ }^{\mathrm{TM}} 293$ Expression Medium, Gibco \#12338) were transiently transfected with the vector coding for the scFv-Fc. The supernatant $(20$ $\mathrm{mg} / \mathrm{L}$ ) was collected after 4 days.
\end{abstract}

Antigen: $5 \times 10^{5}$ D. discoideum $\mathrm{DH} 1$ cells, sedimented on a 22×22 mm glass coverslip (Menzel-Gläser) for 90 minutes at room temperature in HL5 medium, were used.

Protocol: Cells were fixed either $(i)$ with HL5 $+4 \%$ paraformaldehyde (w/v) (Applichem, \#A3013) for $30 \mathrm{~min}$, blocked with $\mathrm{PBS}+40 \mathrm{mM}$ ammonium chloride $\left(\mathrm{NH}_{4} \mathrm{Cl}\right)$
(Applichem, \#A3661) for $5 \mathrm{~min}$, and then permeabilized in PBS $+0.1 \%$ Triton X-100 for $5 \mathrm{~min}$; or (ii) with methanol at $-20{ }^{\circ} \mathrm{C}$ for $2 \mathrm{~min}$. Cells were then washed once (1 min) with PBS, and once (5 min) with PBS $+0.2 \%$ $(\mathrm{w} / \mathrm{v})$ BSA (PBS-BSA). Cells were then incubated for 30 min with the original mouse hybridoma 221-42-1 supernatant (dilution 1:1 in PBS-BSA) or with the AN703 antibody $(5 \mu \mathrm{g} / \mathrm{ml}$ in PBS-BSA). After 3 washes $(5,5,15$ $\mathrm{min}$ ) with PBS-BSA, cells were incubated for $30 \mathrm{~min}$ with secondary goat anti-mouse IgG conjugated to AlexaFluor488 (1:400, Molecular Probes \#A11029). After 3 washes $(5,5,15 \mathrm{~min})$ with PBS-BSA and one wash (5 min) with PBS, coverslips were mounted on slides (Menzel-Gläser, $76 \times 26 \mathrm{~mm}$ ) with Möwiol (Hoechst) $+2.5 \%(\mathrm{w} / \mathrm{v})$ DABCO (Fluka, \#33480). Pictures were taken using a Zeiss LSM700 confocal microscope, with a $63 x$ Neofluar oil immersion objective.

\section{Results}

The AN703 antibody, like the 221-42-1 antibody (Monnat et al., 1997), labels the endoplasmic reticulum of Dictyostelium discoideum (Fig. 1). For both antibodies, cytosolic elements as well as a perinuclear staining are visible (Fig. 1).

\section{References}

Monnat J, Hacker U, Geissler H, Rauchenberger R, Neuhaus EM, Maniak M, Soldati T. Dictyostelium discoideum protein disulfide isomerase, an endoplasmic reticulum resident enzyme lacking a KDEL-type retrieval signal. FEBS Lett. 1997;418(3):357-62. PMID: 9428745.

\section{Conflict of interest}

The authors declare no conflict of interest. 


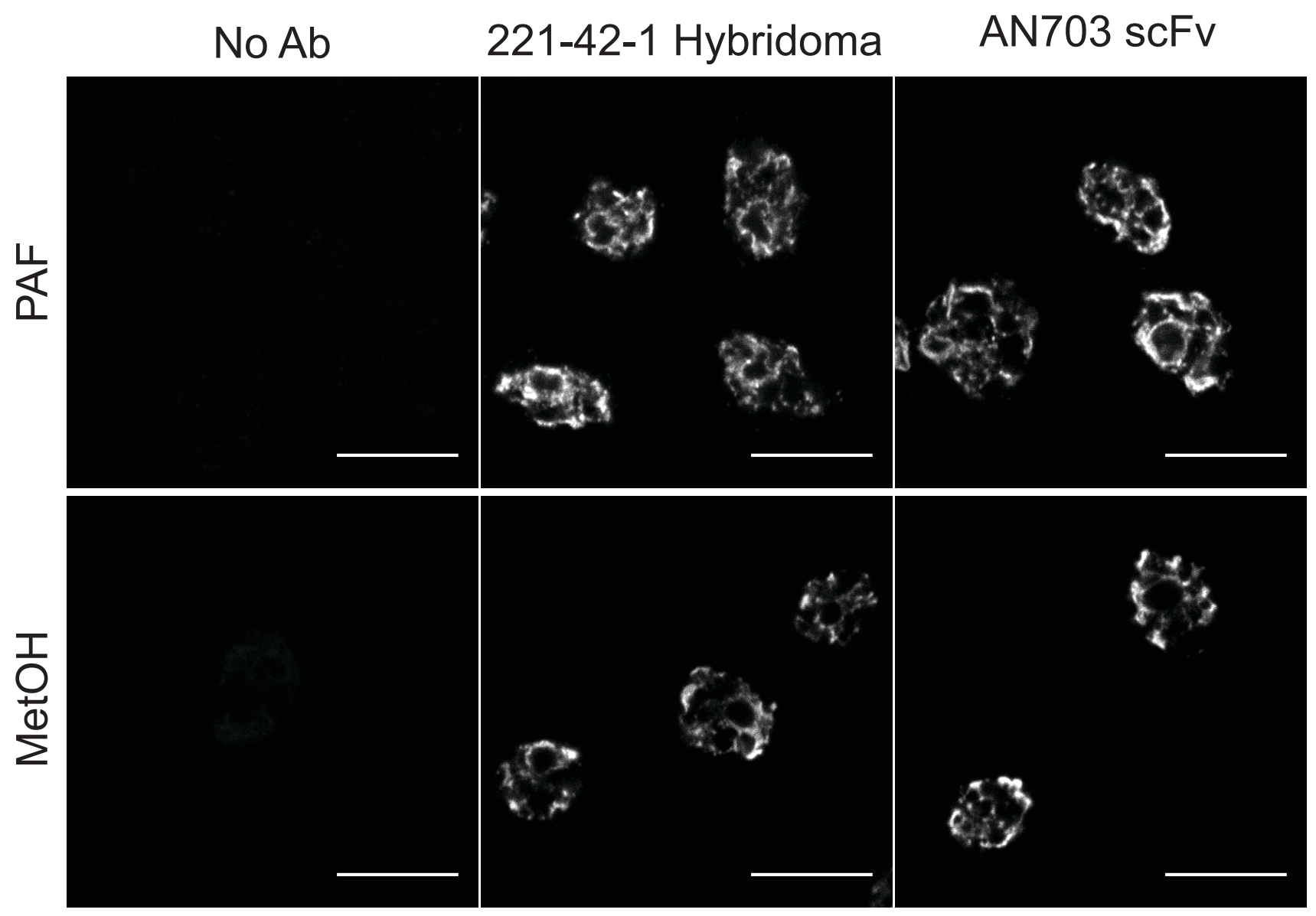

Fig. 1. The 221-42-1 and the AN703 antibodies label the endoplasmic reticulum of Dictyostelium cells, using two different fixation/permeabilization methods. No labelling was seen when the primary antibody was omitted (No Ab). Scale bar: $10 \mu \mathrm{m}$. 